\title{
Primera cita de Archaeatya chacei Villalobos, 1959 (Crustacea, Decapoda, Atyidae) en la Isla de Coiba, Panamá y su distribución en América Central
}

\author{
M. del Real Fernández
}

Archaeatya chacei Villalobos, 1959 es un camarón neotropical de agua dulce de la familia de los Atyidos y la única especie descrita para este género hasta el momento. Fue descrito por primera vez a partir de algunos ejemplares procedentes de la Isla de Cocos, en el Océano Pacífico. Los caracteres morfológicos de esta especie se encuentran entre los géneros Atya y Potimirim, con algunos caracteres relictos caridinianos, lo que impidió a Villalobos (1959) colocarlo dentro de alguno de los géneros existentes de la Familia Atyidae.

La única referencia acerca de la distribución de esta especie fue dada por Chace Jr. (1983) de forma muy superficial y sin especificar las referencias bibliográficas. Hasta el momento su distribución está restringida a la vertiente pacífica de Centroamérica, bajando ligeramente por la costa de Colombia contigua a Panamá.

Archaeatya chacei tiene una distribución principalmente insular: Isla de Cocos en Costa Rica (Villalobos, 1959 y Abele \& Kim, 1984), Archipiélago de las Perlas (Abele \& Blum, 1977) e Isla de Coiba en Panamá. Además aparece en zonas muy concretas de la costa pacífica de Colombia (Ramos et al., 1991) incluyendo el registro de Smalley (1963) en Costa Rica que cita la aparición de algunos individuos de Archaeatya sp. en un pequeño manantial en Dominical, en la cuenca del río Barú, en la Provincia de Puntarenas. Sin embargo en la vertiente continental pacífica de Panamá no fue encontrado por Abele \& Blum (1977).

Los ejemplares estudiados fueron recogidos en arroyos de la Isla de Coiba, Panamá ( $7^{\circ} 30^{\prime}$ Lat. N; $82^{\circ} 20^{\prime}$ Long. O) durante los meses de enero de 1994 y 1996, en el curso de varias expediciones para la realización del inventario de biodiversidad de los invertebrados de agua dulce en el Parque Nacional de Coiba y la costa del Pacífico de Panamá. Fueron capturados con una red de mano con una luz de malla de $160 \mathrm{~mm}$ por el método Kicking (Hynes, 1961), que consiste en remover el fondo del río con los pies situando la red contra corriente, a lo largo de unos 10 metros en el eje longitudinal del arroyo. El material fue depositado en una bandeja y extraídos los ejemplares a mano, conservándolos en alcohol al $70 \%$. La longitud del caparazón (lc) fue medida desde el margen postorbital hasta el extremo posterior dorsal medio del cefalotórax. Así mismo se realizaron mediciones de conductividad (Hanna Instruments, Mod. HI9033), oxígeno disuelto (Yellow Springs Instruments, Mod. YSI 55) y $\mathrm{pH}$ y temperatura (Hanna Instruments, Mod. HI-9025C) en cada lugar de muestreo, así como anotaciones sobre las características del hábitat en que se encontraron. Los ejemplares están depositados en la colección de invertebrados no insectos del Museo Nacional de Ciencias Naturales de Madrid (MNCN).

Se encontró un individuo en el río Playa Hermosa (10-1-94, MNCN 20.04/3728), cinco en la surgencia contigua a las aguas termales (27-1-94, MNCN 20.04/3895, 20.04/3896, 20.04/3894, $20.04 / 3893$ y $20.04 / 3718)$, uno en la quebrada de Catival (7-1-96, MNCN 20.04/3736) y uno en el río Punta Esquina (11-1-96, MNCN 20.04/3697). Fueron estudiados con lupa y microscopio ZEISS.

\section{Archaeatya chacei Villalobos, 1959}

Archaeatya chacei Villalobos 1959: 332, lams. 1-3, fig. 25. Abele \& Blum 1977: 243. Abele \& Kim 1984: 958. Ramos, von Prahl \& Ríos 1991: 56, fig. 1.

* Museo Nacional de Ciencias Naturales. Calle José Gutiérrez Abascal no 2. 28006 Madrid, España. e-mail: monrf1g@pinar2.csic.es. 
Material Examinado.- Segunda quebrada del río Playa Hermosa, Isla de Coiba, leg. A. García-Valdecasas (1 hembra, 10 Enero 1994). Quebrada contigua a las aguas termales, $3 \mathrm{~km}$ al S de Río San Juan, Isla de Coiba, leg. A. García-Valdecasas (2 machos, 2 juveniles y 1 hembra, 27 Enero 1994). Quebrada Catival, Isla de Coiba, leg. A. García-Valdecasas, E. Bello y M. del Real (1 macho, 7 Enero 1996). Río Punta Esquina, Isla de Coiba, leg. A. García-Valdecasas, E. Bello y M. del Real (1 macho, 11 Enero 1996).

MedidAS.- Machos, lc: 2.5-4.0 mm; hembras, lc: $3.50-4.15 \mathrm{~mm}$.

DiAgNOSIS.- Rostro corto y sin espinas sobre ambos márgenes. Ángulo suborbital no agudo, ligeramente obtuso con vértice redondeado y sin diente agudo pterigostomiano. Diente lateral del escafocerito corto y romo. Carpo del primer y segundo pereiópodos de igual longitud que el ancho de la palma de las quelas; ancho de las quelas usualmente mayor que la mitad de su longitud. Dactilo de los tercero y cuarto pereiópodos con dos espinas accesorias a la uña. Endópodo del primer pleópodo del macho con apéndice interno corto, con ganchos retinaculares (Ramos et al., 1991).

HÁBITAT.- Riachuelos y arroyos de aguas dulces $\mathrm{y}$ transparentes de fondos pedregosos o arcillosos y con abundante materia vegetal en descomposición. Viven cercanos a la desembocadura para permitir el desarrollo de las zoeas en el mar, por lo que los arroyos son de bajo caudal, longitud relativamente corta y con desembocadura al mar.

COLOR EN VIDA.- Cuerpo café ocre o verde oscuro, con máculas pequeñas amarillo claro o verde oliva sobre las partes laterales del cefalotórax y abdomen, ojos negros, rostro bermejo (Ramos et al., 1991).

BiologíA.- La alimentación la realiza mediante filtrado con las cerdas de los extremos de los pereiópodos primeros y segundos, y consiste principalmente en fragmentos de plantas acuáticas y en mucha menor medida de restos de integumentos de artrópodos (Abele \& Blum, 1977). La época de reproducción se extiende durante la estación húmeda, aunque se han dado casos de hembras ovígeras durante el inicio de la estación seca (Abele \& Blum, 1977 y Ramos et al., 1991).

En la quebrada Catival el ejemplar fue encontrado en la parte baja del curso, por debajo de los 30 metros de altitud, junto con otros ejemplares de la misma Familia (Atya tenella, Potimirim glabra, Atya sp. y Potimirim sp.), de la Familia Palaemonidae y cangrejos Brachyura. La conductividad media de este arroyo fue de $210 \mathrm{mS}, 6.1$ $\mathrm{mg} / \mathrm{l}$ de oxígeno disuelto y un $\mathrm{pH}$ de 8.1. La anchura máxima del curso era de unos $2 \mathrm{~m}$ y unos $30 \mathrm{~cm}$ de profundidad, y la temperatura del agua y del aire

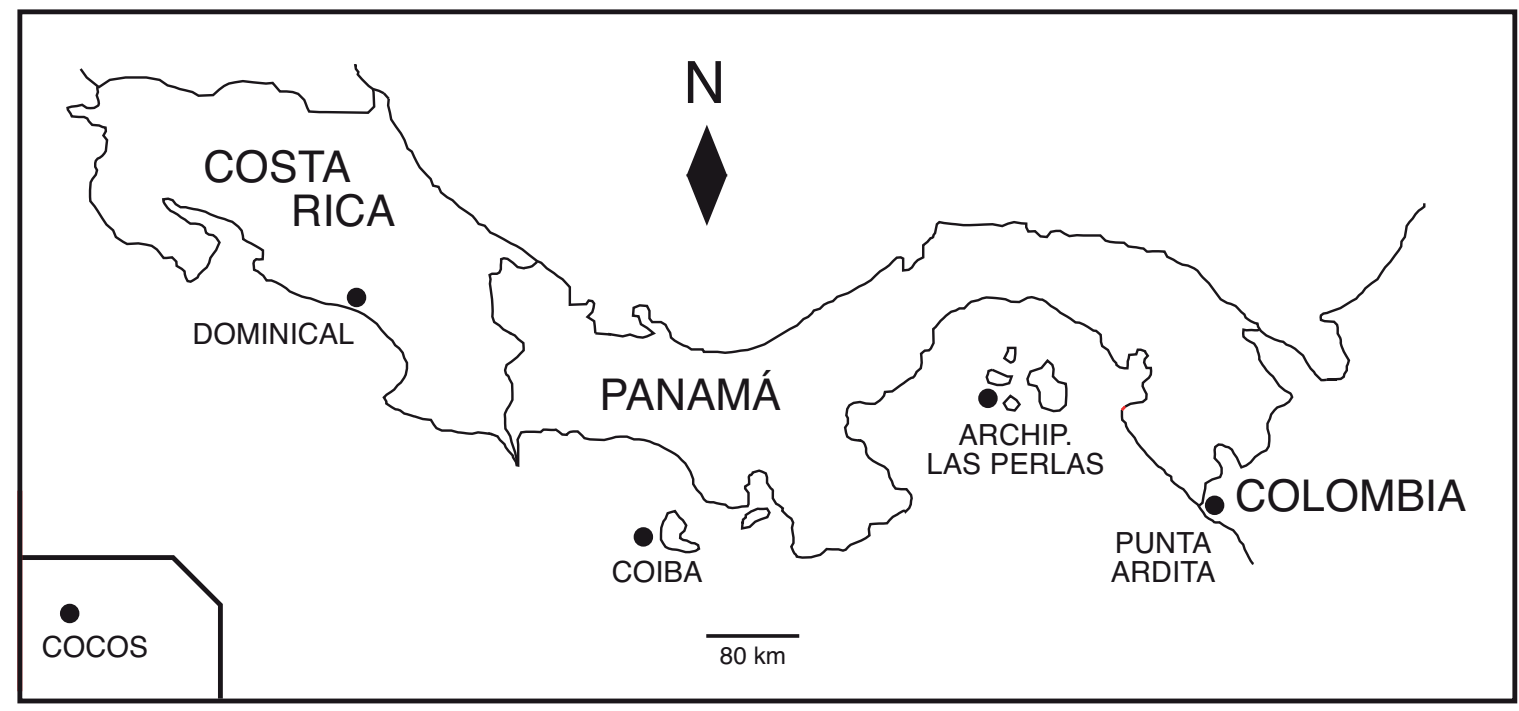

Fig. 1.- Localidades donde ha sido encontrado Archaeatya chacei.

Fig. 1.- Sites were Archaeatya chacei was found. 
de 25 y $26^{\circ} \mathrm{C}$ respectivamente. El fondo con grava gruesa y arena, con abundante materia vegetal en descomposición.

En el río Punta Esquina, en el que solo se muestreó en un punto a unos $20 \mathrm{~m}$ de altitud, se encontró el ejemplar junto a otros de la misma Familia (Atya tenella y Atya sp.), de la Familia Palaemonidae y cangrejos Brachyura. El curso, de unos $35 \mathrm{~m}$ de ancho y $40 \mathrm{~cm}$ de profundidad, estaba cubierto de vegetación y el fondo con arena gruesa y restos vegetales en descomposición. Se registró una temperatura del agua y del aire de 24.2 y $24.9^{\circ} \mathrm{C}$ respectivamente, así como $6.03 \mathrm{mg} / \mathrm{l} \mathrm{de}$ oxígeno disuelto, un $\mathrm{pH}$ de 5.9 y una conductividad de $158.4 \mathrm{mS}$ (García-Valdecasas et al., 1997).

La distribución geográfica de este camarón conocida hasta el momento (fig. 1) indica que tiene unos estados larvales pelágicos de vida corta, que necesitan para su desarrollo unas salinidades altas (Ramos et al., 1991). Estas condiciones tan solo se dan en lugares muy concretos de la costa pacífica de Centro América y son las siguientes: ríos cortos $\mathrm{y}$ arroyos que desembocan directamente al mar para que permitan a los estados larvales pasar de una alta salinidad del mar al agua dulce de los arroyos y la ausencia de grandes ríos cercanos a éstos, de tal forma que se evite la dilución excesiva del agua del mar en la zona costera ya que serviría de barrera para el desarrollo de las zoeas. Así, estas condiciones solo se dan en arroyos de islas con suficiente altitud para tener caudales durante la estación seca (Isla de Cocos, Isla de Coiba y Archipiélago de las Perlas) o en el continente, en zonas costeras con unas importantes elevaciones a corta distancia (Punta Ardita en la Cordillera de Jurado en la frontera entre Colombia y Panamá así como en Dominical, en la Fila Costeña, Costa Rica). Por todo esto, es de esperar que $A$. chacei sólo viva en pequeños arroyos costeros alejados de grandes desembocaduras dentro del continente y en islas.

\section{AGRADECIMIENTOS}

Al Consejo Superior de Investigaciones Científicas (CSIC) y a la Agencia Española de Cooperación Internacional (AECI) por la financiación de las expediciones. A Santiago Castroviejo, del Jardín Botánico de Madrid, por organizar y hacer posible estas expediciones. A Antonio García-Valdecasas y Carolina Noreña Janssen, del Museo Nacional de Ciencias Naturales de Madrid (España), por sus consejos.

\section{Referencias}

ABeLE, L. G. \& Blum, N., 1977. Ecological aspects of the freshwater decapod crustaceans of the Perlas Archipelago, Panamá. Biotropica, 9(4): 239-252.

Abele, L. G. \& KIM, W., 1984. Notes on the freshwater shrimps of Isla del Coco with the description of Macrobrachium cocoense, new species. Proceedings of the Biological Society of Washington, 97(4): 951960.

Chace Jr., F. A., 1983. The Atya-like shrimps of the Indo-Pacific region (Decapoda: Atyidae). Smithsonian Contributions to Zoology, 384: 1-54.

García-Valdecasas, A., CAmacho, A. I., Aranzadi, P. y Bello, E., 1997. Las Aguas dulces de la isla de Coiba (Panamá). 109-125. En Flora y fauna del Parque Nacional de Coiba (Panamá). Inventario preliminar. S. Castroviejo (ed.). Agencia Española de Cooperación Internacional. Madrid. 534 pp.

Hynes, H. B. N., 1961. The invertebrate fauna of a welsh mountain stream. Archiv für Hidrobiologie, 57(3): 344-388.

Ramos, G. E., von Prahl, H. \& Ríos, R., 1991. Los Camarones átidos (Decapoda: Atyidae) de la vertiente del Pacífico de Colombia. Cespedesia, 18(61): 5371.

Smalley, A. E., 1963. The genus Potimirim in Central America. Revista de Biología Tropical, 11: 177-183.

Villalobos, A., 1959. Un nuevo género de Atyidae (Crustacea: Decapoda) procedente de la Isla de Cocos. Anales del Instituto de Biología de la Universidad Autónoma de México, 30(1-2): 331-347. 